\title{
Streptococcal Antibody Probe Crosses the Blood Brain Barrier and Interacts within the Basal Ganglia
}

\author{
Robyn Gebhard, Courtney Huff, Mathew Osborne, Lisa Riegle, Marie Kelly-Worden* \\ Department of Physiology and Health Science, Ball State University, Muncie, USA \\ Email: ${ }^{*}$ mlkellyworde@bsu.edu
}

Received 19 January 2015; accepted 30 March 2015; published 1 April 2015

Copyright (C) 2015 by authors and Scientific Research Publishing Inc.

This work is licensed under the Creative Commons Attribution International License (CC BY). http://creativecommons.org/licenses/by/4.0/

c) (i) Open Access

\begin{abstract}
Within the brain, the basal ganglia (basal nuclei) regulates wanted movement and inhibits unwanted movement. This area of the brain is intertwined with capillary beds that bring nutrients to the brain and form the blood brain barrier. During disease state, antibodies are increased in circulation, and movement of these antibodies into the basal ganglia can occur. Streptococcal infection can lead to the generation of antibodies that have autoimmune activity within the brain. These antibodies have been implicated in neurological disorders. In our laboratory, an in vitro study of a monoclonal mouse antibody generated against the class 1 epitope of the M6 protein has demonstrated binding within the basal ganglia of Lewis rat brains. Here we present an in vivo study using Lewis rats injected with either the streptococcal antibody or an anti-myosin positive control. The interaction and movement of the antibody from blood vessels into the tissues of the basal ganglia was determined through the use of immunofluorescence and fluorescent microscopy and is contrasted with IgG injected and uninjected controls. Our data demonstrates that the streptococcal antibody penetrates the blood brain barrier within 24 hours (as determined by the presence of immunofluorescence outside of blood vessels) and remains significantly elevated above control values even 72 hours after injection $(p<0.05)$. In contrast, the anti-myosin positive control was not visualized in the interstitial fluid until 48 hours post injection and was no longer significantly above control levels by 72 hours. IgG injected controls did not display movement of antibody into the brain. Therefore, the streptococcal antibody is capable of crossing the blood brain barrier and interacting with tissues of the basal ganglia.
\end{abstract}

\section{Keywords}

Blood Brain Barrier, Antibodies, Basal Ganglia, Streptococcus, Rat

\footnotetext{
${ }^{*}$ Corresponding author.
}

How to cite this paper: Gebhard, R., et al. (2015) Streptococcal Antibody Probe Crosses the Blood Brain Barrier and Interacts within the Basal Ganglia. Open Journal of Pathology, 5, 42-49. 


\section{Introduction}

The basal ganglia is composed of the caudate and putamen (the striatum or $\mathrm{CPu}$ ) and globus pallidus, along with the subthalamus and substantia nigra. After receiving information from the cerebral cortex, the basal ganglia facilitates wanted movement while inhibiting unwanted movement [1]. In the movement pathway, the caudate and putamen are normally silent while the globus pallidus sends signals to the thalamus to prevent movement. When a signal arrives from the cortex to the caudate and putamen, it overrides the inhibition of the globus pallidus by ending the inhibitory signal (Figure 1). The result is movement. When an individual is healthy, the normal function of the basal ganglia promotes the individual's daily activities. The blood brain barrier (BBB) normally provides protection against the introduction of chemicals and antibodies that may compromise the function of the basal ganglia. However, during disease state, the BBB may become compromised and; the production of antibodies against one's own tissue that are capable of binding in the basal ganglia may occur. These autoimmune antibodies are implicated in movement disorders including PANDAS, tic disorders and chorea [2] [3].

Antibodies against the basal ganglia may be generated as a legitimate response to bacteria such as a group A streptococcal infection. Streptococcal antibodies are implicated in the pathogenesis of strep infection [4]. For example, streptococcal membrane proteins or $\mathrm{M}$ proteins can induce cross-reactive antibodies against neuronal tissues in humans and in rats [5]. Certain M proteins, such as the M6 protein, have been found to produce antibodies that cross-react with proteins in the basal ganglia more intensely than in cerebellar or cortical tissue [6]. The monoclonal mouse antibody probe, mAb10F5, is a Group A streptococcal M serotype against the M6 protein [7]. This serotype reacts with the class 1 epitope in the conserved $C$ repeat region of the M6 protein and other $\mathrm{M}$ proteins [7] [8]. It is suspected of interacting with myosin in both cardiac and brain tissue. Previous in vitro research in our laboratory has demonstrated that mAb10F5, when applied to brain slices from Lewis rats, bound in regions of the basal ganglia, especially the caudate and putamen. The current study expands upon our previous research and examines whether the antibody can cross the blood brain barrier and, if so, the regions in which binding occurs preferentially. Since the antibody is suspected of binding cardiac myosin, a myosin (Type II) antibody is used as a positive control. IgG2b injected rat brains and uninjected rat brains serve as negative controls.

\section{Methods}

The Lewis rat serves as a current animal model for streptococcal research [9]. In order to examine binding of mAb10F5 in vivo, Lewis rats ( 200 - 325 g) were used. Animals were obtained from the Physiology Lewis rat colony at Ball State University or from the original Lewis rat colony of Harlan Laboratories. Blood samples for serum analysis were obtained prior to initial injection and prior to euthanization. The experimental group consisted of six rats that were injected via the tail vein with $~ 1: 200$ blood volume (based on body weight) of mAb10F5. The experimental control group $(n=6)$ was injected with $\sim 1: 200$ blood volume of myosin Type II antibody (AbDSerotec, Bio-Rad, USA). This anti-myosin mouse antibody is specific for cardiac myosin and has

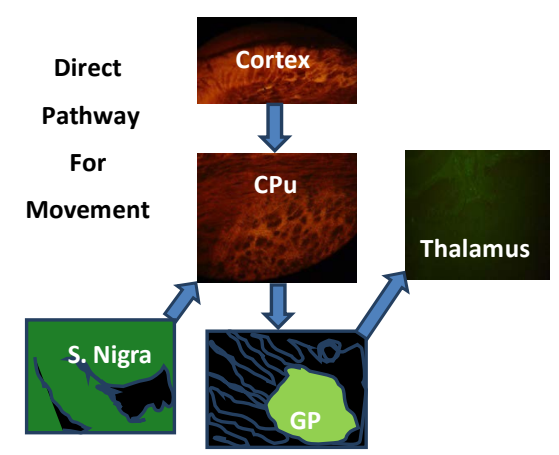

Figure 1. The direct pathway of movement involves the structures of the Basal Ganglia (nucleus); the caudate and putamen $(\mathrm{CPu})$ and the globus pallidus (GP). When a signal arrives to the $\mathrm{CPu}$, it overrides the inhibition of the globus pallidus by ending the inhibitory signal to the thalamus. Transient excitatory inputs to the $\mathrm{CPu}$ are shown in the diagram above from the cortex and substantianigra pars compacta (S. Nigra). 
been shown to bind in rat hearts. The negative controls consisted of IgG2b $(1 \mathrm{mg} / \mathrm{ml})$ injected with $~ 1: 200$ blood volume $(n=6)$ and uninjected $(n=3)$ groups.

Three time points post injection were examined (24, 48, and 72 hours). For each time point, two experimental animals, two experimental control animals and two negative control injected and one negative control (uninjected) were examined. Rats were euthanized at 24 hours, 48 hours, or 72 hours after injection according to group using carbon dioxide followed by a thoracotomy. The brain, heart, liver, and kidneys were all harvested. All procedures were approved by the Animal Care and Use Committee of Ball State University.

\section{Preparation of Brain Tissue}

The brain was rinsed with PBS, and the cerebrum separated and stored in $4 \%$ paraformaldehyde at $4{ }^{\circ} \mathrm{C}$ until use. The cerebrum was then quartered and sliced coronally using a Licor vibratome (90 micron slices) and processed for immunoblotting using blocking buffer (30 minutes), PBS, and anti-mouse Alexa Fluor 488. Briefly, slices were treated with PBS with $0.5 \%$ Triton X-100 and rocked for 30 minutes. The solution was removed, and the slices were washed for ten minutes with PBS (3x). The tissue was blocked with Odyssee blocking buffer (LICOR, USA) for 30 minutes. The tissue was then treated with 1:3000 anti-mouse Alexa Fluor 488 (Invitrogen, Life Technologies, USA) for 90 minutes. The solution was then removed and the slices were washed with PBS (3x). The slices were treated with Sudan black to decrease background fluorescence for 30 minutes, then removed; and the slices were washed with PBS. The slices were examined for the presence of Alexa Fluor 488 using fluorescence microscopy. Photographs were taken under the same conditions for each slice. Mean fluorescence values were obtained using Image Pro 6 software (normalized scale 0 - 255). Mean fluorescent values for slices were averaged together to obtain the final mean fluorescent unit value [10].

\section{Data Analysis}

Lewis rats injected with either Type II cardiac anti-myosin or mAb10F5 were euthanized at 24 hours, 48 hours and 72 hours. The brain was removed and the cerebrum was separated from the cerebellum. The brain was quartered into rostral, mid-rostral, mid-caudal and caudal sections. Mid-rostral sections containing anterior striatal and/or middle striatal capillary beds were sliced and examined for the presence of mouse antibody using Alexa fluor 488 anti-mouse as the secondary antibody. Mean fluorescence values of specific regions; caudate and putamen $(\mathrm{CPu})$, cortex, and hippocampus were determined using the original-digitally captured images and image pro 6 which transformed the picture into a bit map and assigned an intensity for Alex Fluor 488 to each pixel (scale 0 - 255). The average mean values within the area of fluorescence for 2 or more slices, in general, were obtained and used as the mean fluorescence intensity for the given slice region (repeat measurements provided similar results). Only one false-positive slice occurred within the control group and was removed from analysis. Fluorescent unit values were analyzed for significance for each group as compared to either experimental or control groups using single factor ANOVA.

\section{Results}

Upon examination, both antibodies (Type II cardiac anti-myosin and mAb10F5) were found in the region of the caudate and putamen (CPu) at all time points (Figure 2). The level of fluorescence was significant for the mAb10F5 group beginning at 24 hours in the brain. Fluorescence increased at the 48 hours time point and decreased, but remained significant, at the 72 hours time point.

Anti-myosin followed a similar pattern. Significance was observed first at the 24 hours time point, an increase in fluorescence at the 48 hours time point, and a decrease in fluorescence at the 72 hours time point. However, the level of significance dropped at the 72 hours time point as compared to control to just above $p=0.05$ (Table 1).

Alexa Fluor 488 produced a background fluorescence around blood vessels in the rat brain (Figure 2(e)). This background fluorescence can be used to determine the location of cortical arteries and of the capillary beds of the $\mathrm{CPu}$ by allowing one to observe the boundaries of the capillaries. In animals injected with mAb10F5, the boundaries of the capillaries are compromised at 24 hours post injection in the region of the $\mathrm{CPu}$; observed as a cloud of fluorescence that appears outside the boundaries of the capillaries. This compromise is also observed in anti-myosin injected rats, but the cloud does not appear until 48 hours post injection in slices from anti-myosin injected rats (Figure 3). 


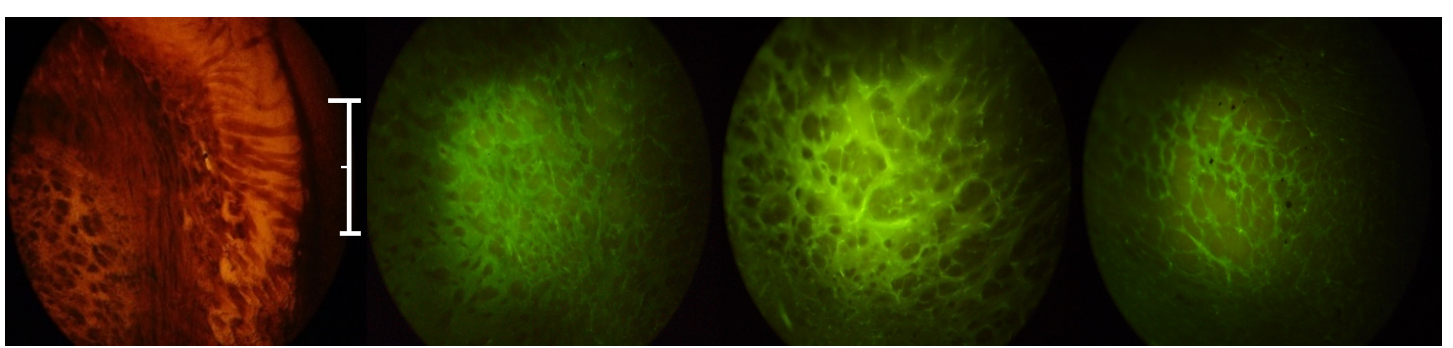

(a)

(b)

(c)

(d)

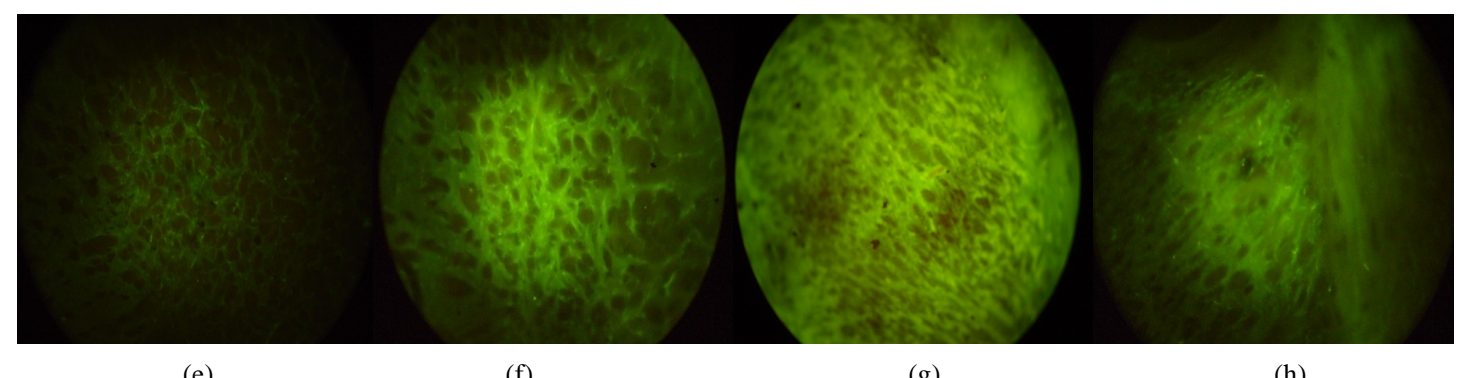

(e)

(f)

(g)

(h)

Figure 2. Both myosin (Type II) antibody and mAb10F5 bound in the caudate/putamen; CPu. Fluorescent microscopy using a $10 \times 0.25$ NA CP-Achromat objective in midrostral CPu with Alexa Fluor 488 secondary. (a) Brain slice in the coronal plane demonstrating the border between cortex and the $\mathrm{CPu}$ of the rat. Bar in A equals $\sim 1 \mathrm{~mm}$; (b) 24 hours myosin (Type II) antibody; (c) 48 hours myosin (Type II) antibody; (d) 72 hours myosin (Type II) antibody; (e) Negative control; (f) 24 hours mAb10F5; (g) 48 hours mAb10F5; and (h) 72 hours mAb10F5 near the CPu border.

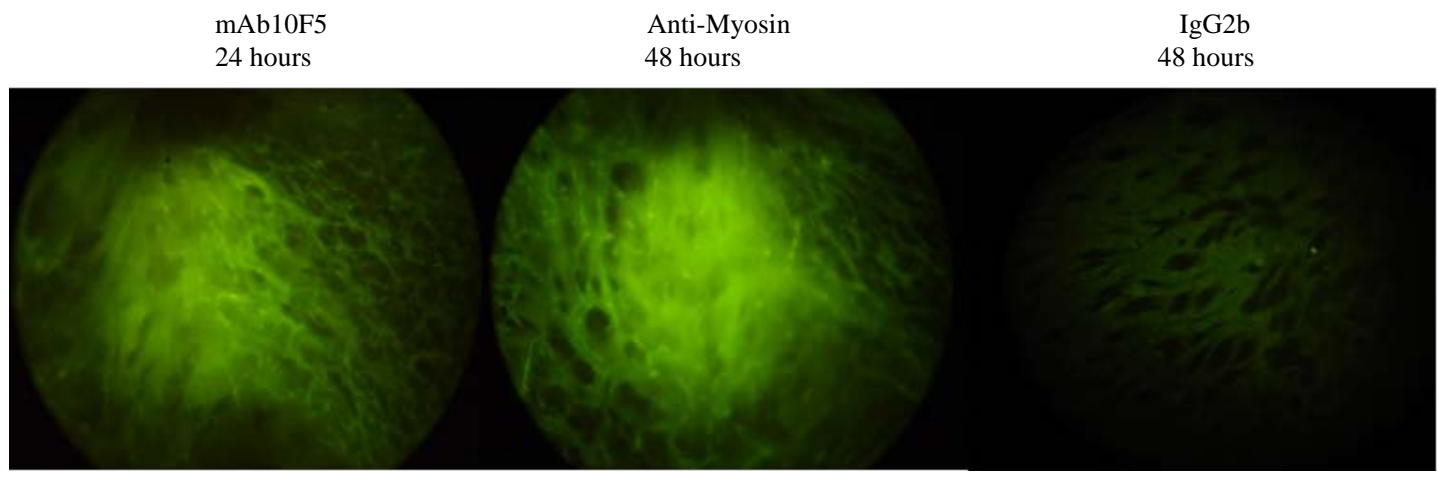

(a)

(b)

(c)

Figure 3. In the midrostral $\mathrm{CPu}$, a cloud of fluorescence (Alexa Fluor 488) indicating the presence of antibody was observed in the mAb10F5 treated rats at 24 hours (a) and in the myosin (Type II) antibody treated rats at 48 hours (b). This phenomena were not observed in IgG2b injected negative controls (c).

Table 1. Average Mean Fluorescent values for control, mAb10F5, myosin (Type II) antibody (anti-myosin) at 24 hours, 48 hours, and 72 hours time points.

\begin{tabular}{ccc}
\hline & Average Mean Fluorescence \pm SE & p-Value \\
\hline Negative Control & $62.807 \pm 9.94$ & \\
24 Hours mAb10F5 & $124.018 \pm 12.79$ & 0.00437 \\
Anti-Myosin & $95.087 \pm 19.07$ & 0.03953 \\
48 Hours mAb10F5 & $132.255 \pm 7.44$ & 0.00044 \\
Anti-Myosin & $153.522 \pm 13.80$ & 0.00040 \\
72 Hours mAb10F5 & $117.961 \pm 10.81$ & 0.01132 \\
Anti-Myosin & $100.087 \pm 14.53$ & 0.05876 \\
\hline
\end{tabular}

Fluorescent levels previously reported as Midrostral CPu (Robyn Gebhard, Honors Thesis, 2009, Ball State University) are presented as values in the table on a scale from 0 - 255 with standard error (SE) added. p-values reported were obtained from a single factor ANOVA, $\mathrm{p}<0.05$ for significance. 
Other regions of the brain were examined to determine if there was preference in binding. The hippocampus is a region of interest in the brain due to its role in the conversion of short-term to long-term memory. Damage to the hippocampus is often observed in stroke, and an ischemic event could promote movement of antibody into the hippocampus due to compromise of the BBB. However, investigation of the hippocampus provided negative results. Both the mAb10F5 and anti-myosin antibody treated groups were negative for all time points as compared with the controls (Figure 4). Data was analyzed using a single factor ANOVA from fluorescent images taken with a $5 \times$ objective as these represented each group at every time point (Table 2). Data obtained for the images taken with the 10× objective were also negative. The hippocampus for the 48 and 72 hours mAb10F5, and

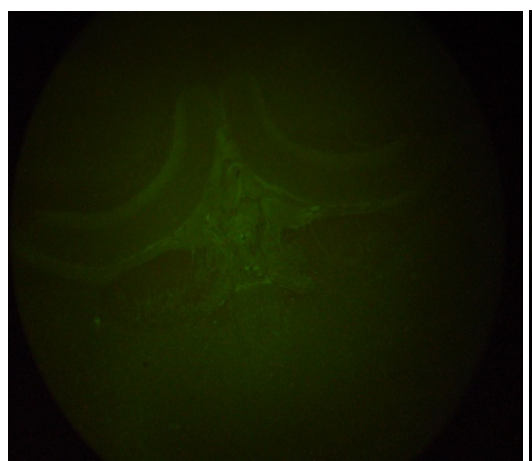

(a)

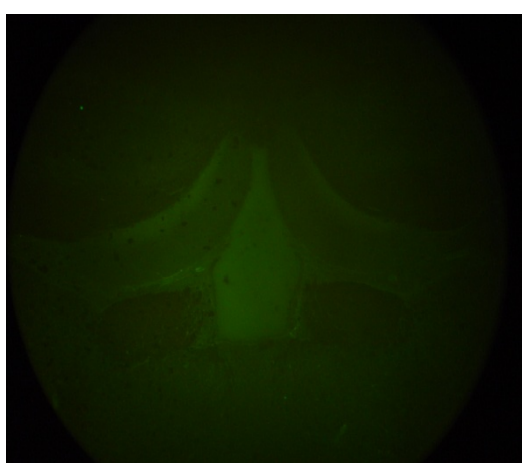

(b)

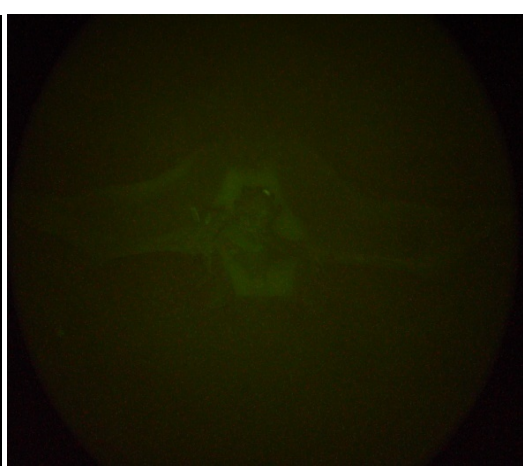

(c)

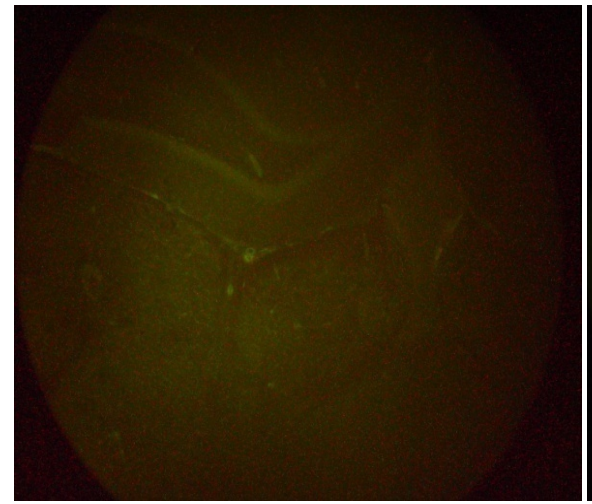

(d)

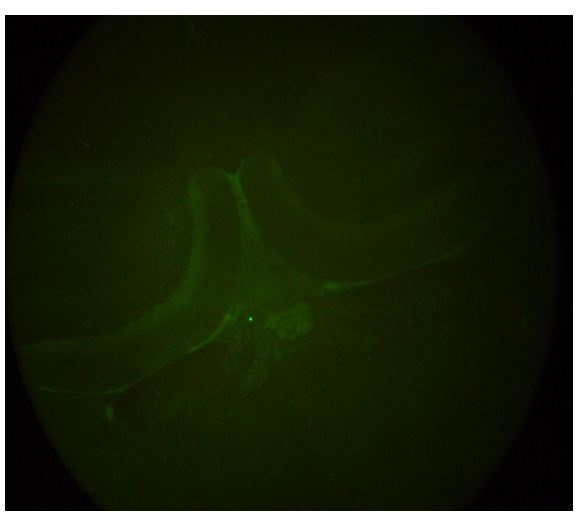

(e)

Figure 4. Both antibodies (myosin (Type II) antibody and mAB10F5) failed to bind significantly in the hippocampus. Fluorescent images taken with a $5 \times 0.12$ NA CP-Achromat objective showing the negative hippocampus. (a) Negative control; (b) 48 hours myosin (Type II) antibody; (c) 72 hours myosin (Type II) antibody; (d) 48 hours mAb10F5; (e) 72 hours mAb10F5.

Table 2. Average Mean Fluorescence levels are shown for the midrostral cortex and hippocampus (hippo) in the chart.

\begin{tabular}{|c|c|c|c|c|}
\hline \multirow{2}{*}{ Cortex (Midrostral) } & \multicolumn{2}{|c|}{ Average Mean Fluorescence } & \multicolumn{2}{|c|}{ p Value } \\
\hline & Cortex \pm SE & Hippo \pm SE & Cortex & Hippo \\
\hline Negative Control & $72.70 \pm 12.64$ & $52.20 \pm 2.14$ & & \\
\hline 24 Hours mAb10F5 & $76.39 \pm 16.06$ & $55.96 \pm 3.32$ & 0.86363 & 0.43055 \\
\hline Anti-Myosin & $69.29 \pm 15.00$ & $39.02 \pm 4.38$ & 0.88508 & 0.02748 \\
\hline 48 Hours mAb10F5 & $86.16 \pm 35.95$ & $45.01 \pm 3.20$ & 0.65631 & 0.03310 \\
\hline Anti-Myosin & $66.29 \pm 9.96$ & $50.90 \pm 4.51$ & 0.71387 & 0.43526 \\
\hline 72 Hours mAb10F5 & $52.78 \pm 4.28$ & $34.19 \pm 5.20$ & 0.28929 & 0.01501 \\
\hline Anti-Myosin & $69.52 \pm 2.31$ & $45.30 \pm 2.63$ & 0.85706 & 0.07418 \\
\hline
\end{tabular}

All values were insignificant ( $\mathrm{p}>0.05$ ) when compared by ANOVA to the negative control group for the cortex. Fluorescence levels obtained for the hippocampus were often below control values and contained at least 1 Average Mean Fluorescent data set. p-values were obtained from a single factor ANOVA (significance at $\mathrm{p}>0.05$ ). 
the 24 hours anti-myosin antibody-treated groups were significantly negative $(\mathrm{p}<0.05)$ when compared to controls.

The cortex serves as a regulator of the basal ganglia and as a pathway for removal of protein from the CPu. Therefore, we investigated the level of fluorescence observed in the cortex of our rats (Figure 5). Data from images in the same area of the midrostral cortex of antibody-treated rats was compared to controls (Table 2). Values were taken from within the cortical tissue, and external cortical blood vessel fluorescence was ignored (see Figure 5). The cortex of the uninjected control group had an average fluorescence of 72.70. The mAb10F5 treated group had an average fluorescence in the cortex of 76.39 at 24 hours, 86.16 at 48 hours, and 52.78 at 72 hours. The anti-myosin antibody group had displayed even lower fluorescence values in the cortex (69.29 at 24 hours, 66.29 at 48 hours, and 69.52 at 72 hours). Therefore, neither the mAb10F5 group nor the anti-myosin (Type II) antibody group was significant for fluorescence in the cortex at any time point when compared to negative controls.

\section{Discussion}

The streptococcal mouse antibody probe mAb10F5 provides relevant information for human disease state since M serotypes associated with the majority of acute rheumatic fever outbreaks possess the epitope (Class I) defined by monoclonal antibody probes 10B6 and 10F5 [7] [11]. Possession of antibodies that react with this epitope does not by itself conclude that the antibody will find a way into the brain and promote an autoimmune reaction. However, increased permeability of the blood brain barrier can occur during disease state. Pro-inflammatory and pro-angiogenic factors can mediate blood-brain barrier dysfunction [12].

The streptococcal antibody mAb10F5 presented significantly positive fluorescence in the midrostral region of the caudate and putamen $(\mathrm{CPu})$ of the basal ganglia, but not in the cortex or hippocampus (which was significantly negative). The specificity of binding in this region is consistent with in vitro data from our laboratory and original findings of Husby et al., 1976, that placed human streptococcal antibodies here [13]. The natural blood supply of the CPu may provide an answer to the mystery of how the antibodies may enter into the brain here. In rats, as in humans, vascular supply of the CPu is derived from two main sources: branches of the middle cerebral artery and of the anterior cerebral artery. The Lateral Striatal Artery (LSA) branches from the middle cerebral artery and provides capillary beds. Lesions involving the medial or lateral LSA result primarily in motor and motor-task performance deficits [14]. Blood moves slower through the capillaries, allowing more time for antibody interactions.

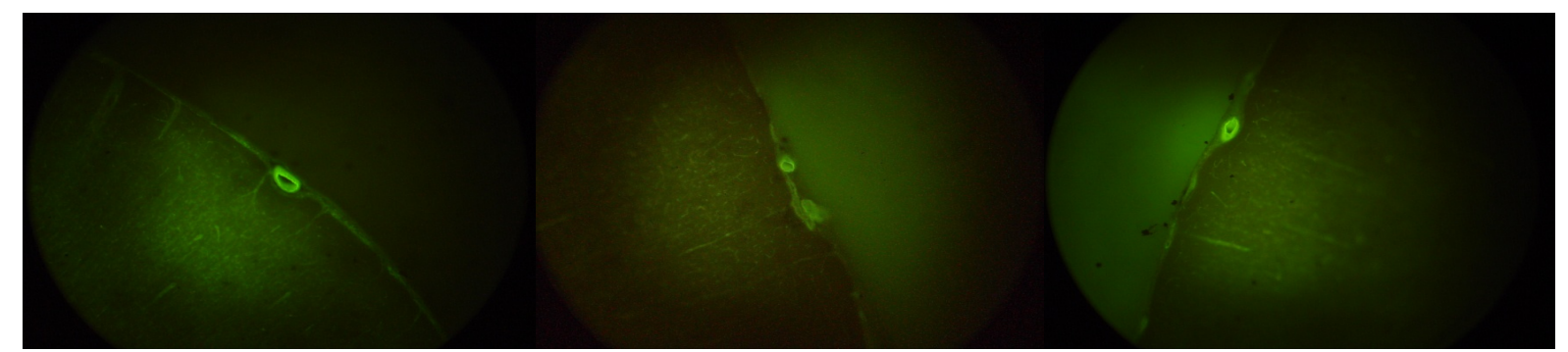

(a)

(b)

(c)

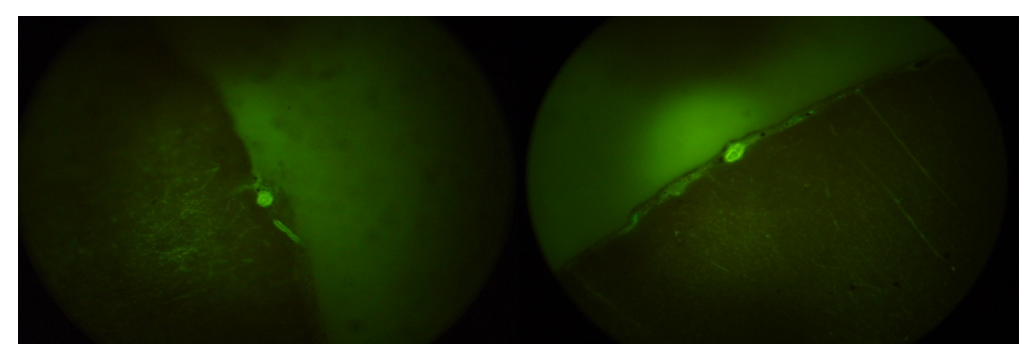

(d)

(e)

Figure 5. Cortical regions displayed fluorescence (Alexa Fluor 488 treated tissue) of cortical blood vessels that did not differ significantly from controls. (a) Negative control; (b) 48 hours myosin (Type II) antibody; (c) 72 hours myosin (Type II) antibody; (d) 48 hours mAb10F5; and (e) 72 mAb10F5. 
Clearance of antibodies from the rat bloodstream by the liver may occur rapidly during the first hour following injection [15]. However, saturation is reached and some antibody remains circulating in the blood. In fact, it may be days after injection of mouse IgG antibody into the rat bloodstream before complete removal occurs [16]. Given this, the presence of the antibody in blood vessels 48 - 72 hours after injection is not surprising. However, the level of both mAb10F5 and the anti-myosin antibodies, as measured by fluorescence intensity, in the brain tissue was surprising and suggests that the Blood Brain Barrier (BBB) only provided minimal protection from these antibodies. Removal of antibody from the brain was consistent with what has been observed previously for albumin. The half-life for removal of albumin from rat caudate is 10 - 12 hours [17]. Within 24 hours after injection of albumin into the $\mathrm{CPu}$, it is found on the glial limitans and pial lining as well as in the extracellular space of cortical neuropil [18]. This is somewhat consistent with our findings that the anti-myosin antibody was no longer significant in the CPu 24 hours after being observed outside of the capillaries. However, we do not observe a significant difference from controls in the cortex 24 hours later (at the 72 hours time point) as might be expected.

Polymorphic cells known as pericytes exist at the blood brain barrier. Pericytes are actin and myosin containing cells that are situated closest to brain endothelial cells morphologically and share a common basement membrane [19]. In the rat brain, pericytes cover approximately 1/4 of capillary outer surfaces. Pericytes are responsible for the regulation of activity of the endothelial cells of the capillaries, mediate inflammation, and control of capillary like structure formation and capillary diameter. Thus, these cells play a role in the maintenance of the BBB and brain homeostasis [20]. In the lipopolysaccharide model of septic encephalopathy, pericyte detachment and microglial activation is proposed to play a role in mediation of BBB disruption. Disorganization in the pericyte and basal lamina unit correlate with increased microglial activation and cerebrovascular permeability [21]. Thus, an interaction of antibodies with pericyte myosin may indirectly promote cerebral permeability and the movement of antibody into the brain. There is also a second explanation for the ability of mAb10F5 to find a way across the BBB. The antibody may be crossing directly across the plasma membrane of capillary endothelial cells and entering into the CPu where autoimmune interactions then take place. The antibody has displayed reactivity with lipids in vitro and our data suggests that it is similar to anti-phosphatidylserine in behavior [22] [23].

The mechanism of entry into the brain appears to be point related disruption of the blood brain barrier with the capillary beds of the basal ganglia being affected the most as is seen by clouds of antibody appearing in localized regions. The ability of these probes to cross the blood brain barrier in the region of the basal ganglia provides insight into the ability of anti-myosin antibodies to find a way into the brain. Our study indicates that these antibodies are removed from the initial region of entry (the $\mathrm{CPu}$ ), as is observed by the decrease in fluorescence at the 72 hours time point for both antibodies and the loss of significance for anti-myosin. It will be interesting to see if prolonged exposure to antibody, as is the case with maintained release, would lead to a buildup of antibody in the brain and the potential for plaque formation, inflammation, or movement disorders. These will be topics for future investigation.

\section{Acknowledgements}

We would like to thank Dr. Vincent Fischetti for providing such a large supply of mAb10F5.

\section{References}

[1] Mink, J.W. (2003) The Basal Ganglia and Involuntary Movements: Impaired Inhibition of Competing Motor Patterns. Archives of Neurology, 60, 1365-1368. http://dx.doi.org/10.1001/archneur.60.10.1365

[2] Church, A.J., Dale, R.C. and Giovannoni, G. (2004) Anti-Basal Ganglia Antibodies: A Possible Diagnostic Utility in Idiopathic Movement Disorders? Archives of Disease in Childhood, 89, 611-614. http://dx.doi.org/10.1136/adc.2003.031880

[3] Martino, D., Church, A.J., Dale, R.C. and Giovannoni, G. (2004) Antibasal Ganglia Antibodies and Their Relevance to Movement Disorders. Current Opinion in Neurology, 17, 425-432. http://dx.doi.org/10.1097/01.wco.0000137532.76491.19

[4] Kirvan, C.A., Swedo, S.E., Kurahara, D. and Cunningham, M.W. (2006) Streptococcal Mimicry and Antibody-Mediated Cell Signaling in the Pathogenesis of Sydenham's Chorea. Autoimmunity, 39, 21-29. http://dx.doi.org/10.1080/08916930500484757 
[5] Dale, R.C. (2003) Autoimmunity and the Basal Ganglia: New Insights into Old Diseases. QJM, 96, 183-191. http://dx.doi.org/10.1093/qjmed/hcg026

[6] Bronze, M.S. and Dale, J.B. (1993) Epitopes of Streptococcal M Proteins That Evoke Antibodies That Cross-React with Human Brain. Journal of Immunology, 151, 2820-2828.

[7] Jones, K.F., Khan, S.A., Erickson, B.W., Hollingshead, S.K., Scott, J.R. and Fischetti, V.A. (1986) Immunochemical Localization and Amino Acid Sequences of Crossreactive Epitopes within the Group A Streptococcal M6 Protein. Journal of Experimental Medicine, 164, 1226-1238. http://dx.doi.org/10.1084/jem.164.4.1226

[8] Jones, K.F. and Fischetti, V.A. (1988) The Importance of the Location of Antibody Binding on the M6 Protein for Opsoniation and Phagocytosis of Group A M6 Streptococci. Journal of Experimental Medicine, 167, 1114-1123. http://dx.doi.org/10.1084/jem.167.3.1114

[9] Li, Y., Heuser, J.S., Kosanke, S.D., Hemric, M. and Cunningham, M.W. (2004) Cryptic Epitope Identified in Rat and Human Cardiac Myosin S2 Region Induces Myocarditis in the Lewis Rat. Journal of Immunology, 172, 3225-3234. http://dx.doi.org/10.4049/jimmunol.172.5.3225

[10] Kelly-Worden, M., Hammer, L., Gebhard, R., Schrader, L., Griffin, M. and Cooper, D. (2014) ANA Positive Serum from SLE Patients Promotes Cardiovascular and CNS Manifestations in the Lewis Rat. Journal of Pharmacy and Bioallied Science, 6, 198-204. http://dx.doi.org/10.4103/0975-7406.135247

[11] Quinn, A., Ward, K., Fischetti, V.A., Hemric, M. and Cunningham, M.W. (1989) Immunological Relationship between the Class I Epitope of Streptococcal M Protein and Myosin. Infection and Immunity, 66, 4418-4424.

[12] Choi, Y.K. and Kim, K.W. (2008) Blood-Neural Barrier: Its Diversity and Coordinated Cell-to-Cell Communication. BMB Reports, 41, 345-352.

[13] Husby, G., Van De Rijn, I., Zabriskie, J.B., Abdin, Z.H. and Williams Jr., R.C. (1976) Antibodies Reacting with Cytoplasm of Subthalamic and Caudate Nuclei Neurons in Chorea and Acute Rheumatic Fever. Journal of Experimental Medicine, 144, 1094-1110. http://dx.doi.org/10.1084/jem.144.4.1094

[14] Feekes, J.A. and Cassell, M. (2006) The Vascular Supply of the Functional Compartments of the Human Striatum. Brain, 129, 2189-2201. http://dx.doi.org/10.1093/brain/awl158

[15] Pardridge, W.M., Buciak, J.L. and Friden, P.M. (1991) Selective Transport of an Anti-Transferrin Receptor Antibody through the Blood-Brain Barrier in Vivo. Journal of Pharmacology and Experimental Therapeutics, 259, 66-70.

[16] Klibanov, A.L., Martynov, A.V., Slinkin, M.A., Sakharov, I.Y., Smirnov, M.D., Muzykantov, V.R., Danilov, S.M. and Torchilin, V.P. (1988) Blood Clearance of Radiolabeled Antibody: Enhancement by Lactosamination and Treatment with Biotin-Avidin or Anti-Mouse IgG Antibodies. Journal of Nuclear Medicine, 29, 1951-1956.

[17] Szentistvanyii, I., Patlak, C.S., Ellis, R.A. and Cserr, H.F. (1984) Drainage of Interstitial Fluid from Different Regions of Rat Brain. American Journal of Physiology. Renal Physiology, 246, F835-F844.

[18] Ohata, K. and Marmarou, A. (1992) Clearance of Brain Edema and Macromolecules through the Cortical Extracellular Space. Journal of Neurosurgery, 77, 387-396. http://dx.doi.org/10.3171/jns.1992.77.3.0387

[19] Nakagawa, S., Deli, M.A., Kawaguchi, H., Shimizudani, T., Shimono, T., Kittel, A., Tanaka, K. and Niwa, M. (2009) A New Blood-Brain Barrier Model Using Primary Rat Brain Endothelial Cells, Pericytes and Astrocytes. Neurochemistry International, 54, 253-263. http://dx.doi.org/10.1016/j.neuint.2008.12.002

[20] Bernacki, J., Dobrowolska, A., Nierwińska, K. and Małecki, A. (2008) Physiology and Pharmacological Role of the Blood-Brain Barrier. Pharmacological Reports, 60, 600-622.

[21] Nishioku, T., Dohgu, S., Takata, F., Eto, T., Ishikawa, N., Kodama, K.B., Nakagawa, S., Yamauchi, A. and Kataoka, Y. (2009) Detachment of Brain Pericytes from the Basal Lamina Is Involved in Disruption of the Blood-Brain Barrier Caused by Lipopolysaccharide-Induced Sepsis in Mice. Cellular and Molecular Neurobiology, 29, 309-316.

[22] Kelly-Worden, M., Eisa, A., Huff, C. and Osborne, M. (2013) Streptococcal Anti-Phospholipid Antibody, mAB10F5, Binds Greatest in the Cardiac Valve Region. Biophysical Journal, 104, 154a. http://dx.doi.org/10.1016/j.bpj.2012.11.872

[23] Kelly-Worden, M., Osborne, M. and Gebhard, R. (2012) mAb10F5 Is an Anti-Phospholipid Antibody that Binds in the Basal Ganglia in Vivo. Biophysical Journal, 102, 496a. http://dx.doi.org/10.1016/j.bpj.2011.11.2718 